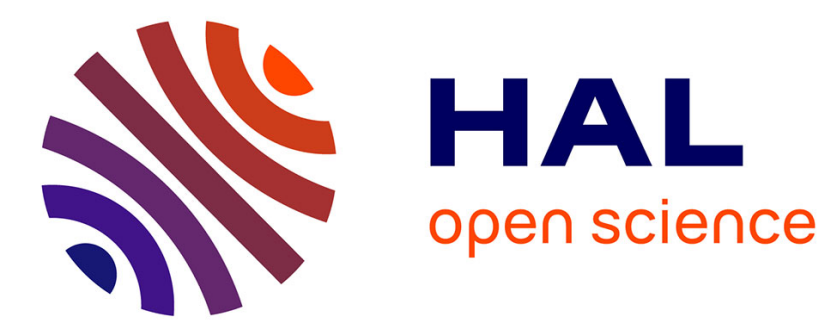

\title{
A Projection Approach to the Numerical Analysis of Limit Load Problems
}

Guillaume Carlier, Myriam Comte, Ioan R. Ionescu, Gabriel Peyré

\section{To cite this version:}

Guillaume Carlier, Myriam Comte, Ioan R. Ionescu, Gabriel Peyré. A Projection Approach to the Numerical Analysis of Limit Load Problems. Mathematical Models and Methods in Applied Sciences, 2011, 21 (6), pp.1291-1316. hal-00450000

\section{HAL Id: hal-00450000 https://hal.science/hal-00450000}

Submitted on 24 Jan 2010

HAL is a multi-disciplinary open access archive for the deposit and dissemination of scientific research documents, whether they are published or not. The documents may come from teaching and research institutions in France or abroad, or from public or private research centers.
L'archive ouverte pluridisciplinaire HAL, est destinée au dépôt et à la diffusion de documents scientifiques de niveau recherche, publiés ou non, émanant des établissements d'enseignement et de recherche français ou étrangers, des laboratoires publics ou privés. 


\title{
A Projection Approach to the Numerical Analysis of Limit Load Problems
}

\author{
Guillaume Carlier \\ CEREMADE \\ Université Paris Dauphine \\ carlier@ceremade.dauphine.fr \\ Ioan R. Ionescu \\ LPMTM \\ Université Paris 13 \\ ioan.r.ionescu@gmail.com
}

\author{
Myriam Comte \\ Laboratoire Jacques-Louis Lions, \\ Université Pierre et Marie Curie \\ comte@ann.jussieu.fr \\ Gabriel Peyré \\ CEREMADE \\ Université Paris Dauphine \\ peyre@ceremade.dauphine.fr
}

January 24, 2010

\begin{abstract}
This paper proposes a numerical scheme to approximate the solution of (vectorial) limit load problems. The method makes use of a strictly convex perturbation of the problem, which corresponds to a projection of the deformation field under bounded deformation and incompressibility constraints. The discretized formulation of this perturbation converges to the solution of the original landslide problem when the amplitude of the perturbation tends to zero. The projection is computed numerically with a multi-steps gradient descent on the dual formation of the problem.
\end{abstract}

Keywords: limit load analysis, functions of bounded deformation, penalizations, Nesterov algorithm.

\section{Introduction}

Limit analysis, which is the simplest approach for modeling the inelastic response of structures, is based on a very idealized representation of a rigid, perfectly plastic material subjected to slowly increasing loads. The main problem in limit analysis is to find the maximum multiple of the force distribution that the solid can withstand without collapsing. Usually, the associated collapse flow field exhibits discontinuities on some surfaces and the strain rates are bounded measures (strain localization). That is why, from both mathematical and numerical points of view, limit analysis was and remains a difficult problem.

The numerical methods in limit analysis are based on the discretization of the kinematic or static variational principles (established in [18]) using finite element method techniques and on convex and linear programming. The first results were obtained in [5, 23, 24], while the literature on FE methods applied to limit analysis is very extensive (see for instance $[4,13,14,30,32,42])$. Recently, a new method, called discontinuous velocity domain splitting (DVDS) and originated in [25] (see also [22]), was proposed in [26]. DVDS is a mesh free method which focuses on the strain localization and completely neglects the bulk deformations. 
The goal of this paper is to propose a new numerical technique for the limit load problem, based on a projection with a specific weighted $\mathrm{L}^{2}$ norm. Only the Von-Mises yield condition is considered in this paper, but the proposed numerical method may, in principle, be extended to other plastic models. The main idea comes from a convergence result of a perturbed problem, obtained in [7] in the anti-place case, which selects the maximal collapse domain (called the Cheeger set in a geometrical context [12] and related to the first eigenvalue of the degenerate 1-Laplacian operator $[17,28]$ ). The associated numerical method was developed in [9] using a finite difference discretization and a subgradient projection iterative algorithm, introduced in [15].

The Cheeger constant was introduced in [12] to give a lower bound on the first eigenvalue of the Laplacian and found a wide range of applications (denoising model in image processing, continuous max-flow/min-cut duality) in recent years [35, 3, 2, 36, 37]. For convex domains, the uniqueness of the Cheeger set can be proved $[10,1]$ proved and explicit constructions (see [29]) are known. The connection between limit load analysis and the Cheeger problem with weights was established in $[25,22]$ where various properties of the minimizers can be found. The existence of Cheeger sets were proved, but uniqueness does not hold in general which makes it difficult to compute numerically Cheeger sets. Fortunately, there is a unique maximal Cheeger set (see [8]) and it is proved in [7] that it can be approximated by adding a small strictly convex penalization. Starting from this penalization scheme, a convergent projection algorithm is implemented in [9] to approximate maximal Cheeger sets.

Our aim in this paper is to generalize the strategy and the convergence results of [9] to handle the, physically more relevant, two-dimensional in-plane flow case that is detailed in the next paragraph. The extension of the method proposed in [9] for the anti-plane flow to the vectorial case of the in-plane or of 3-D problems is a difficult task. It presents several additional difficulties, both from theoretical and numerical points of view. First of all, the coarea formula is not any more valid in the vectorial case and the link between the variational and the geometrical problems is an open problem. Second, we have to handle the divergence free condition and the space of bonded deformations BD (instead of $B V$ ), difficulties which which are not present in the anti-plane (scalar) case.

Let us outline the content of the paper. In section 2 we give the physical description of the limit load problem. The initial variational formulation is relaxed to get a good functional framework for the existence of a solution. In section 3 we introduce the antiplane and in-plane cases. For the first of them (scalar case) we recall the main theoretical and numerical results, needed to understand the approach of the in-plane (vectorial) case developed in the remainder of the paper. In section 4 we present the penalization scheme. We prove the convergence of the penalization scheme and the convergence for the discretized problems. Section 5 describes a numerical scheme to solve the discretized limit load problem. This scheme solves an unconstrained dual optimization problem using an accelerated first order algorithm. Finally, numerical simulations for three important limit load problems (two notched tensile, indentation and porous metals) are presented and some caparisons with analytical and numerical results are given. 


\section{Problem statement}

\subsection{Physical description}

Let $\mathcal{D} \subset \mathbb{R}^{3}$, be the domain occupied by a rigid-plastic body. We shall denote by $\boldsymbol{\sigma}: \mathcal{D} \rightarrow \mathcal{S}_{3}$, (where $\mathcal{S}_{3}$ denotes the space of $3 \times 3$ symmetric matrices), the Cauchy stress tensor which is in equilibrium under the the body forces $\boldsymbol{b}$ and the applied forces $\boldsymbol{f}$ :

$$
\operatorname{div} \boldsymbol{\sigma}+\boldsymbol{b}=0 \quad \text { in } \mathcal{D}, \quad \boldsymbol{\sigma} \boldsymbol{n}=\boldsymbol{f} \quad \text { on } \Gamma,
$$

where $\boldsymbol{n}$ is the outward unit normal to $\Gamma=\partial \mathcal{D}$.

At each point $x \in \mathcal{D}$, we consider the admissible set of stresses $K=K(x)$ which will be supposed to be a convex and closed subset of $\mathcal{S}_{3}$ with $0 \in K$. This set is expressed through the yield potential $F: \mathcal{D} \times \mathcal{S}_{3} \rightarrow \mathbb{R}$ and the yield limit $\kappa$ :

$$
K(x)=\left\{\boldsymbol{\tau} \in \mathcal{S}_{3} ; F(x, \boldsymbol{\tau}) \leqslant \kappa(x)\right\} .
$$

We will restrict ourselves in this paper to one of the most used yield criterium, defined through the von-Mises yield potential

$$
F(x, \boldsymbol{\tau})=\left|\boldsymbol{\tau}-\frac{\operatorname{trace}(\boldsymbol{\tau})}{3} \boldsymbol{I}\right|
$$

and the yield limit $\kappa(x)=\sqrt{2} g(x)$. The rigid-plastic constitutive equation (flow rule) relates the rate of deformation tensor

$$
\boldsymbol{D}=\boldsymbol{D}(\boldsymbol{v}):=\frac{1}{2}\left(\boldsymbol{\nabla} \boldsymbol{v}+\nabla^{t} \boldsymbol{v}\right) \text { i.e. } \boldsymbol{D}(\boldsymbol{v})_{i j}=\frac{\partial_{i} \boldsymbol{v}_{j}+\partial_{j} \boldsymbol{v}_{i}}{2}, 1 \leqslant i, j \leqslant 3,
$$

associated to the velocity field $\boldsymbol{v}: \mathcal{D} \rightarrow \mathbb{R}^{3}$, to the stress tensor $\boldsymbol{\sigma}$ through

$$
\boldsymbol{D}(\boldsymbol{v})(x) \in \partial \Phi(x, \boldsymbol{\sigma}(x)) \quad \text { in } \mathcal{D},
$$

where $\Phi(x, \cdot)$ is the indicator function of the convex $K(x)$ and $\partial \Phi(x,$.$) denotes the sub-$ gradient of $\Phi(x,$.$) .$

The dual formulation of the flow rule can be expressed by using the conjugate $\Phi^{*}$ of the indicator function $\Phi$, called the strain rate potential:

$$
\boldsymbol{\sigma}(x) \in \partial \Phi^{*}(x, \boldsymbol{D}(\boldsymbol{v})(x)) \quad \text { in } \mathcal{D} .
$$

For the von-Mises model, the expression of $\Phi^{*}$ is simple to compute

$$
\Phi^{*}(x, \boldsymbol{D})=\left\{\begin{array}{ccc}
\kappa(x)|\boldsymbol{D}|, & \text { if } & \operatorname{trace}(\boldsymbol{D})=0, \\
+\infty, & \text { if } & \operatorname{trace}(\boldsymbol{D}) \neq 0 .
\end{array}\right.
$$

The model is supplemented by a boundary condition on velocity. For the sake of simplicity, we will only consider here the homogeneous Dirichlet condition:

$$
\boldsymbol{v}=0 \quad \text { on } \Gamma_{V}
$$

where $\Gamma_{V}$ is a fixed part of $\partial \mathcal{D}$. 


\subsection{Variational formulation}

In order to give the (kinematic) variational formulation of the rigid-plastic problem we introduce $\Pi$, the plastic dissipation power and $P$, the power of external loads:

$$
\Pi(\boldsymbol{u}):=\int_{\mathcal{D}} \Phi^{*}(x, \boldsymbol{D}(\boldsymbol{u})(x)) d x, \quad P(\boldsymbol{u}):=\int_{\mathcal{D}} \boldsymbol{b} \cdot \boldsymbol{u} d x+\int_{\Gamma} \boldsymbol{f} \cdot \boldsymbol{u} d \Gamma .
$$

Note that, in the von-Mises model, the plastic dissipation power is finite only for divergencefree velocity fields ( $\Phi^{*}$ being infinite on matrices with non-zero trace). At least formally (the precise functional framework and the correct expression for $\Pi$ will be detailed in the next paragraph), note that the equilibrium conditions (1), the flow rule (5) and the Dirichlet condition (7) are nothing but the Euler-Lagrange equation for the minimization of $\Pi-P$ over the set $\mathcal{V}$ of divergence-free vector fields that satisfy (7). The (kinematic) variational formulation of the rigid-plastic problem derived from (1), (5), (6) and (7) can therefore be written as the convex optimization problem

$$
\boldsymbol{v} \in \mathcal{V}, \quad \Pi(\boldsymbol{u})-\Pi(\boldsymbol{v}) \geqslant P(\boldsymbol{u}-\boldsymbol{v}), \quad \text { for all } \boldsymbol{u} \in \mathcal{V} .
$$

The limit load problem is then defined as follows. Let us assume that the linear form $P$ of the power of external forces corresponds to a loading process starting from vanishing forces, i.e. we put $P(\boldsymbol{u})=: t L(\boldsymbol{u})$, for all $\boldsymbol{u} \in \mathcal{V}$, where $t \geqslant 0$ is a loading non-dimensional parameter and $L$ is a loading direction. If $\boldsymbol{f}=t \boldsymbol{f}_{0}$ and $\boldsymbol{b}=t \boldsymbol{b}_{0}$ then

$$
L(\boldsymbol{u}):=\int_{\mathcal{D}} \boldsymbol{b}_{0} \cdot \boldsymbol{u} d x+\int_{\Gamma} \boldsymbol{f}_{0} \cdot \boldsymbol{u} d \Gamma .
$$

The limit analysis problem consists in finding the largest loading parameter $t$ for which $\boldsymbol{v} \equiv 0$ is a solution of (9). If we replace $\boldsymbol{v} \equiv 0$ in (9) then we get $\Pi(\boldsymbol{u}) \geqslant t L(\boldsymbol{u})$, for all $\boldsymbol{u} \in \mathcal{V}$. We define now the safety factor or the limit load as

$$
\lambda=: \inf _{\boldsymbol{u} \in \mathcal{V}, L(\boldsymbol{u})=1} \Pi(\boldsymbol{u}) .
$$

Then, $t \leqslant \lambda$ if and only if the rigid-plastic structure $\mathcal{D}$ can stand the load $t L$, (i.e. $\boldsymbol{v} \equiv 0$ is a solution of (9)). For $t>\lambda$, the collapse of the structure is expected.

Let us finally notice that the above limit analysis problem can be rewritten as

$$
\frac{1}{\lambda}=\sup _{\boldsymbol{u} \in \mathcal{V}, \Pi(\boldsymbol{u}) \leqslant 1} L(\boldsymbol{u})
$$

\subsection{Relaxation and mathematical preliminaries}

Our aim now is to give a rigorous meaning to the variational problem (12). For the sake of completeness, we will set the framework in $\mathbb{R}^{d}$ with $d \geqslant 2$ rather than in $\mathbb{R}^{3}$. Let $\mathcal{D}$ be a bounded and $C^{1}$ domain of $\mathbb{R}^{d}$ with $\Gamma:=\partial \mathcal{D}$. Denoting by $\mathcal{M}^{d \times d}=\mathcal{M}^{d \times d}(\mathcal{D})$ the space of bounded $d \times d$-matrix valued Radon measures, the space BD of functions of bounded deformation is by definition

$$
\mathrm{BD}=\mathrm{BD}(\mathcal{D}):=\left\{\boldsymbol{u} \in L^{1}\left(\mathcal{D}, \mathbb{R}^{d}\right): \boldsymbol{D}(\boldsymbol{u}) \in \mathcal{M}^{d \times d}\right\}
$$

with

$$
\boldsymbol{D}(\boldsymbol{u}):=\frac{1}{2}\left(\boldsymbol{\nabla} \boldsymbol{u}+\nabla^{t} \boldsymbol{u}\right) \text {, i.e. } \boldsymbol{D}(\boldsymbol{u})_{i j}=\frac{\partial_{i} \boldsymbol{u}_{j}+\partial_{j} \boldsymbol{u}_{i}}{2}, 1 \leqslant i, j \leqslant d
$$


As usual, BD is equipped with the norm:

$$
\|\boldsymbol{u}\|_{\mathrm{BD}}:=\|\boldsymbol{u}\|_{L^{1}(\Omega)}+\sum_{1 \leqslant i, j \leqslant d}\left\|\boldsymbol{D}(\boldsymbol{u})_{i j}\right\|_{\mathcal{M}} .
$$

For any $d \times d$ matrices $A$ and $B$ we shall denote

$$
|A|^{2}:=\sum_{1 \leqslant i, j \leqslant d} A_{i j}^{2}, A: B:=\sum_{1 \leqslant i, j \leqslant d} A_{i j} B_{i j} .
$$

For $\boldsymbol{u} \in \mathrm{BD}$, we recall that the bounded Radon measure $|\boldsymbol{D}(\boldsymbol{u})|$ is defined by

$$
|\boldsymbol{D}(\boldsymbol{u})|(\omega)=\sup \left\{\frac{1}{2} \sum_{1 \leqslant i, j \leqslant d} \int_{\Omega} \partial_{i}\left(\varphi_{i j}+\varphi_{j i}\right) \boldsymbol{u}_{j}: \varphi \in C_{c}^{1}\left(\omega, \mathbb{R}^{d \times d}\right),|\varphi| \leqslant 1\right\}
$$

for every open $\omega \subset \mathcal{D}$. Of course, one can equivalently define the strong topology of BD by the norm $\boldsymbol{u} \mapsto\|\boldsymbol{u}\|_{L^{1}}+|\boldsymbol{D}(\boldsymbol{u})|(\mathcal{D})$. It is well-known that BD $\subset L^{d /(d-1)}$ with continuous imbedding, that the imbedding $\mathrm{BD} \subset L^{p}$ is compact for $p \in[1, d /(d-1))$ and that BD functions have an $L^{1}(\Gamma)$ trace (see [41] and [38]). As usual, the weak convergence of a sequence $\left(\boldsymbol{u}_{n}\right)_{n}$ in $\mathrm{BD}$ to some $\boldsymbol{u}$ in BD (simply denoted $\boldsymbol{u}_{n} \rightarrow \boldsymbol{u}$ ) means by definition that $\left(\boldsymbol{u}_{n}\right)_{n}$ converges strongly to $\boldsymbol{u}$ in $L^{1}$ and that $\left(\boldsymbol{D}\left(\boldsymbol{u}_{n}\right)\right)_{n}$ converges weakly star in $\mathcal{M}^{d \times d}$ to $\boldsymbol{D}(\boldsymbol{u})$. Let us also recall that a sequence $\left(\boldsymbol{u}_{n}\right)_{n}$ in BD is said to converge for the intermediate topology to some $\boldsymbol{u}$ in $\mathrm{BD}$, if $\boldsymbol{u}_{n} \rightarrow \boldsymbol{u}$ and $\left|\boldsymbol{D}\left(\boldsymbol{u}_{n}\right)\right|(\mathcal{D}) \rightarrow\left|\boldsymbol{D}\left(\boldsymbol{u}_{n}\right)\right|(\mathcal{D})$. Finally, we will extend, when necessary, functions $\boldsymbol{v} \in$ BD by 0 outside $\overline{\mathcal{D}}$ such an extension being of course in $\mathrm{BD}\left(\mathbb{R}^{d}\right)$.

Given some subset $\Gamma_{V}$ of $\Gamma$, a continuous and positive yield limit $\kappa$, define the weight function $g$ by $\sqrt{2} g:=\kappa$, body forces $\boldsymbol{b}_{0}$ defined on $\mathcal{D}$ and boundary traction forces $\boldsymbol{f}_{0}$, the limit analysis problem reads as

$$
\frac{1}{\lambda}:=\sup _{\boldsymbol{v} \in \mathcal{V}}\left\{L(\boldsymbol{v}): \int_{\mathcal{D}} \kappa d|\boldsymbol{D}(\boldsymbol{v})| \leqslant 1\right\}
$$

where

$$
L(\boldsymbol{v}):=\int_{\Omega} \boldsymbol{b}_{0} \cdot \boldsymbol{v}+\int_{\Gamma} \boldsymbol{f}_{0} \cdot \boldsymbol{v}
$$

and $\mathcal{V}$ is the space of all $\boldsymbol{v} \in \mathrm{BD}$ such that

$$
\operatorname{div}(\boldsymbol{v})=0 \text { in } \mathcal{D}^{\prime}(\mathcal{D}), \boldsymbol{v}=0 \text { on } \Gamma_{V} .
$$

We also define

$$
\Gamma_{S}:=\Gamma \backslash \Gamma_{V}
$$

Before we make precise assumptions on the data $\Gamma_{V}, g($ or $\kappa), \boldsymbol{b}, \boldsymbol{f}$, let us indicate that it is well-known that (13) does not in general admit solutions due to the fact that the trace map is not continuous with respect to weak convergence of BD (but it is for the intermediate topology), hence there is no guarantee that one can pass to the limit neither in the boundary term in $L$ nor in the boundary condition $\boldsymbol{v}=0$ on $\Gamma_{V}$. To overcome this difficulty (very similar to what may happen in minimal surfaces problems), Temam and Strang showed in [40] that (13) admits a relaxed formulation that can be defined as follows. From now on, we make the following assumptions: 
- (H1) Either $\Gamma_{V}=\emptyset$ or $\Gamma_{V}$ is nonempty and open in $\Gamma$ and there is an open bounded nonempty subset of $\mathbb{R}^{d}, \Omega^{\prime}$, such that

$$
\mathcal{D} \cap \Omega^{\prime}=\emptyset, \overline{\mathcal{D}} \cap \overline{\Omega^{\prime}}=\overline{\Gamma_{V}}
$$

and

$$
\Omega_{0}:=\mathcal{D} \cup \Gamma_{V} \cup \Omega^{\prime} \text { is open } .
$$

- (H2) $g \in C\left(\bar{\Omega}_{0}, \mathbb{R}\right)$ and $g>0$ on $\bar{\Omega}_{0}$,

- (H3) $\boldsymbol{b}_{0} \in L^{d}(\mathcal{D})$ and the boundary traction is of the form $\boldsymbol{f}_{0}:=f_{0} \boldsymbol{n}$ where $\boldsymbol{n}$ denotes the exterior normal (in other words, the boundary traction is normal) and $f_{0}$ is the trace on $\Gamma$ of a $W^{1, d}$ function (again denoted $f$ ) such that $f_{0}=0$ on $\Gamma_{V}$. One then has for all $\boldsymbol{v} \in \mathrm{BD}$,

$$
L(\boldsymbol{v})=\int_{\Omega} \boldsymbol{b}_{0} \cdot \boldsymbol{v}+\int_{\Gamma_{S}} f_{0} \boldsymbol{v} \cdot \boldsymbol{n}, \quad \text { where } \Gamma_{S}:=\Gamma \backslash \Gamma_{V} .
$$

Let us then define $E$ as

$$
E:=\left\{\boldsymbol{v} \in \mathrm{BD}: \operatorname{div}(\boldsymbol{v})=0 \text { in } \mathcal{D}^{\prime}(\mathcal{D}), \boldsymbol{v} \cdot \boldsymbol{n}=0 \text { on } \Gamma_{V}\right\} .
$$

Note that in $E$ only the normal part of the initial Dirichlet condition (7) is conserved. It is easy to see that $\boldsymbol{v} \in \mathrm{BD}$ belongs to $E$ if and only if

$$
\int_{\mathcal{D}} \boldsymbol{v} \cdot \nabla \varphi=0, \forall \varphi \in C^{1}(\overline{\mathcal{D}}): \varphi_{\mid \Gamma_{S}}=0
$$

so that $E$ is weakly closed in BD. Note also that one can take $W^{1, d}$ test-functions above to characterize $E$.

For every $\boldsymbol{v} \in E$ (extended by 0 outside $\overline{\mathcal{D}}$ ) let us set

$$
\Pi(\boldsymbol{v}):=\int_{\Omega_{0}} \kappa d|\boldsymbol{D}(\boldsymbol{v})|
$$

it follows from the weak star lower semicontinuity of measures of open sets that $\Pi$ is weakly lower semicontinuous. It is moreover classical to check that for all $\boldsymbol{v} \in E$ one has

$$
\begin{aligned}
\Pi(\boldsymbol{v}) & =\int_{\mathcal{D}} \kappa d|\boldsymbol{D}(\boldsymbol{v})|+\frac{1}{2} \int_{\Gamma_{V}} \kappa|\boldsymbol{v} \otimes \boldsymbol{n}+\boldsymbol{n} \otimes \boldsymbol{v}| \\
& =\int_{\mathcal{D}} \kappa d|\boldsymbol{D}(\boldsymbol{v})|+\frac{1}{\sqrt{2}} \int_{\Gamma_{V}} \kappa|\boldsymbol{v}| \\
& =\int_{\mathcal{D}} \kappa d|\boldsymbol{D}(\boldsymbol{v})|+\int_{\Gamma_{V}} g|\boldsymbol{v}| .
\end{aligned}
$$

Assumption (H3) guarantees the weak continuity of $L$ on $E$; indeed for $\boldsymbol{v} \in E$, one has

$$
\int_{\Gamma} \boldsymbol{f}_{0} \cdot \boldsymbol{v}=\int_{\Gamma} f_{0} \boldsymbol{v} \cdot \boldsymbol{n}=\int_{\Gamma_{S}} f_{0} \boldsymbol{v} \cdot \boldsymbol{n}=\int_{\mathcal{D}} \nabla f_{0} \cdot \boldsymbol{v} .
$$

It follows from the results of Temam and Strang [40], that the relaxation of (13) (see theorem 1 for a precise statement) takes the form

$$
\left(\mathcal{P}_{0}\right) \sup \{L(\boldsymbol{u}): \boldsymbol{u} \in E, \Pi(\boldsymbol{u}) \leqslant 1\} .
$$


As seen above, our assumptions guarantee weak lower semicontinuity properties of the previous problem. However, when $\Gamma_{V}$ is empty, a further natural compatibility assumption is required if we want the previous value to be finite. Let us denote by $\mathcal{R}$ the set of rigid motions, that is the set of maps of the form $x \in \mathcal{D} \mapsto A x+b$ with $A$ skew-symmetric and $b \in \mathbb{R}^{d}$, when $d=2$, this set reduces to maps $x \mapsto \lambda x^{\perp}+c$ with $(\lambda, c) \in \mathbb{R} \times \mathbb{R}^{2}$. Since, when $\Gamma_{V}=\emptyset$, for every $\boldsymbol{r} \in \mathcal{R}$ and every $\boldsymbol{v} \in E, \boldsymbol{v}+\boldsymbol{r}$ is admissible, the finiteness of the value of (14) obviously requires that if $\boldsymbol{r} \in \mathcal{R}$ then $L(\boldsymbol{r})=0$. Hence, in the case where $\Gamma_{V}=\emptyset$, we shall also need the following :

(H4) in the case where $\Gamma_{V}=\emptyset, L(\boldsymbol{r})=0$ for every $\boldsymbol{r} \in \mathcal{R}$.

The next relaxation statement then follows from the classical results of Temam and Strang [40]:

Theorem 1. Under the previous assumptions, the supremum in $\left(\mathcal{P}_{0}\right)$ is achieved and:

$$
\frac{1}{\lambda}=\max \left(\mathcal{P}_{0}\right)
$$

Proof. Assume first that $\Gamma_{V}$ is nonempty and satisfies (H1). Let us prove that the supremum in $\left(\mathcal{P}_{0}\right)$ is achieved (the relaxation statement being proved in [40]). Let $\left(\boldsymbol{u}_{n}\right)_{n}$ be some maximizing sequence for $\left(\mathcal{P}_{0}\right)$. It is easy to check that if $\boldsymbol{r} \in \mathcal{R}$ and $\Pi(\boldsymbol{r})=0$ then $\boldsymbol{r}=0$; we thus deduce from proposition 2.3 in [41] that the semi-norm $\Pi$ is equivalent to the BD norm on $E$ so that $\left(\boldsymbol{u}_{n}\right)$ is bounded in BD. Up to a (not relabeled) subsequence, we may assume that there is some $\boldsymbol{u} \in \mathrm{BD}$ such that $\boldsymbol{u}_{n} \rightarrow \boldsymbol{u}$. By weak lower semicontinuity of $\Pi, \Pi(\boldsymbol{u}) \leqslant 1$ moreover $\boldsymbol{u} \in E$ and $L\left(\boldsymbol{u}_{n}\right) \rightarrow L(\boldsymbol{u})$ so that $\boldsymbol{u}$ solves $\left(\mathcal{P}_{0}\right)$.

The previous proof carries over to the case $\Gamma_{V}=\emptyset$ provided (H4) holds. Indeed, in this case, one obtains existence of maximizers for $\left(\mathcal{P}_{0}\right)$ by using Proposition 2.4 in [41] which implies that given a maximizing sequence $\left(\boldsymbol{u}_{n}\right)_{n}$ for $\left(\mathcal{P}_{0}\right)$, one can find a sequence of rigid motions $\boldsymbol{r}_{n}$ such that $\left(\boldsymbol{u}_{n}-\boldsymbol{r}_{n}\right)_{n}$ (which is still a maximizing sequence) is bounded in BD.

Defining:

$$
\mathrm{LD}:=\left\{\boldsymbol{v} \in L^{1}: \boldsymbol{D}(\boldsymbol{v}) \in L^{1}\right\},
$$

in the sequel, we shall also need the following density result (which will imply density in energy of smooth functions)

Proposition 1. Let us further assume that $\mathcal{D}$ is of class $C^{2}$ and that either $\Gamma_{V}$ or $\Gamma_{S}$ is empty, then, for every $\boldsymbol{u} \in E$, there exists a sequence $\left(\boldsymbol{u}_{n}\right) \in E \cap C^{1}(\mathcal{D}) \cap \mathrm{LD}$ that converges to $\boldsymbol{u}$ for the intermediate topology and strongly in $L^{d^{\prime}}$ with $d^{\prime}=d /(d-1)$. We thus have:

$$
\max \left(\mathcal{P}_{0}\right)=\sup \left\{L(\boldsymbol{v}): \boldsymbol{v} \in E \cap C^{1}(\mathcal{D}) \cap \mathrm{LD}, \Pi(\boldsymbol{v}) \leqslant 1\right\} .
$$

Proof. It follows from Theorem 3.4 and remark 3.5 in [39], that there exists a sequence $\left(\boldsymbol{w}_{n}\right)$ in $C^{\infty}(\mathcal{D}) \cap \mathrm{LD}$ that converges to $\boldsymbol{u}$ both strongly in $L^{d^{\prime}}$ and for the intermediate topology and such that $\boldsymbol{w}_{n}=\boldsymbol{u}$ on $\Gamma$ (in the sense of traces) and such that

$$
\operatorname{div}\left(\boldsymbol{w}_{\mathrm{n}}\right) \rightarrow 0 \text { strongly in } \mathrm{L}^{2}
$$

let then $\varphi_{n}$ be the solution of

$$
\left\{\begin{array}{l}
\Delta \varphi_{n}=\operatorname{div}\left(\boldsymbol{w}_{\mathrm{n}}\right) \text { in } \mathcal{D}, \\
\varphi_{n}=0 \text { on } \Gamma_{S}, \\
\nabla \varphi_{n} \cdot \boldsymbol{n}=\boldsymbol{w}_{n} \cdot \boldsymbol{n}=0 \text { on } \Gamma_{V} .
\end{array}\right.
$$


By standard elliptic regularity (since here we have either purely Dirichlet or purely Neumann boundary conditions), we have $\varphi_{n} \rightarrow 0$ in $H^{2}$ and in particular $\left|\mathcal{D}\left(\nabla \varphi_{n}\right)\right| \rightarrow 0$ strongly in the sense of measures, hence the sequence

$$
\boldsymbol{u}_{n}:=\boldsymbol{w}_{n}-\nabla \varphi_{n}
$$

belongs to $E$ and satisfies the desired claim. The identity (15) easily follows : let $\boldsymbol{u} \in E$ be such that $\Pi(\boldsymbol{u}) \leqslant 1$ and let $\boldsymbol{u}_{n}$ be a sequence in $E \cap C^{1}(\mathcal{D}) \cap$ LD that converges to $\boldsymbol{u}$ for the intermediate topology. Then by the continuity of $L$ for this topology, we have $L\left(\boldsymbol{u}_{n}\right) \rightarrow L(\boldsymbol{u})$. Now for $\delta>0$, for large enough $n$, we deduce from the continuity of $\Pi$ with respect to the intermediate topology, that $\boldsymbol{u}_{n} /(1+\delta)$ is admissible for $\left(\mathcal{P}_{0}\right)$ and then

$$
\begin{aligned}
L(\boldsymbol{u}) & =(1+\delta) \lim _{n} L\left(\frac{\boldsymbol{u}_{n}}{1+\delta}\right) \\
& \leqslant(1+\delta) \sup \left\{L(\boldsymbol{v}): \boldsymbol{v} \in E \cap C^{1}(\mathcal{D}) \cap \mathrm{LD}, \Pi(\boldsymbol{v}) \leqslant 1\right\} .
\end{aligned}
$$

The claim thus simply follows from letting $\delta \rightarrow 0^{+}$and taking the supremum with respect to $\boldsymbol{u}$.

Let us remark that when $\Gamma_{V}$ and $\Gamma_{S}$ are nonempty, then it is not true in general that the solution $\varphi_{n}$ of the mixed problem above is $H^{2}$ up to the boundary. Nevertheless, when $d=2$ and $\mathcal{D}$ is a rectangle (with sides parallels to the canonical axes, say), and $\Gamma_{V}$ consists of its vertical (or horizontal) sides, then the previous argument works (it is enough to proceed by reflexion and invoke standard elliptic regularity). This is a relevant variant, since we will treat numerically this case.

\section{The anti-plane and the in-plane cases}

In this section, we focus on two particular two-dimensional (one scalar and vectorial respectively) cases, namely, the anti-plane case (which as, recalled below, is tightly related to the, purely geometric, Cheeger problem) and the in-plane case.

\subsection{The anti-plane case and Cheeger sets}

In the case of a unidirectional- or anti-plane-flow, the domain is of the form $\mathcal{D}=\Omega \times \mathbb{R}$ where $\Omega$ is a bounded domain in $\mathbb{R}^{2}$ with a smooth boundary $\partial \Omega$ divided into parts $\Gamma_{0}, \Gamma_{1}$ such that $\Gamma_{V}=\Gamma_{0} \times \mathbb{R}, \Gamma_{S}=\Gamma_{1} \times \mathbb{R}$. The flow is in the $O x_{3}$ direction i.e. $\boldsymbol{v}=(0,0, u)$ and does not depend on $x_{3}$ so that the incompressibility condition $\operatorname{div}(\boldsymbol{v})=0$ is satisfied. Supposing that $\boldsymbol{f}=0$ and since the non-vanishing components of the rate deformation tensor $\boldsymbol{D}(\boldsymbol{v})$ are $D_{13}=D_{31}=\partial_{1} u / 2, D_{23}=D_{32}=\partial_{2} u / 2$, we get that the limit load can be written in this case as

$$
\lambda=\inf _{u \in \mathrm{BV}, l(u)=1} \pi(u), \quad \pi(u)=\int_{\Omega} g d|\nabla u|+\int_{\Gamma_{0}} g|u|
$$

where $l(u):=\int_{\Omega} b(x) u(x) d x$ and $b$ denotes the component of the forces $\boldsymbol{b}_{0}$ in the $O x_{3}$ direction. If $b$ and $g$ are positive and continuous functions, one can reduce the minimization to nonnegative $u$ 's. If, in addition $\Gamma_{0}=\partial \Omega$, then it follows from the coarea formula that the minimization of the Rayleigh quotient $\pi(u) / l(u)$ may be reduced to characteristic functions of sets of finite perimeter:

$$
\lambda:=\inf _{u \in \mathrm{BV}} \frac{\pi(u)}{l(u)}=\inf _{A \subset \bar{\Omega}} \frac{\int_{\partial A} g}{\int_{A} b} .
$$


For $b=g=1$, the previous problem is known as Cheeger's problem [12]. Subsets of $\bar{O}$ that minimize the ratio perimeter over area are called Cheeger sets and the minimal value of this ratio is known as the Cheeger constant of $\Omega$. The Cheeger constant was introduced in [12] to give a lower bound on the first eigenvalue of the Laplacian. Interestingly, Cheeger's problem has found a wide range of applications in recent years. For instance, Cheeger sets arise quite naturally from the analysis of the Rudin-Osher-Fatemi [35] denoising model in image processing and in the minimizing total variation flow [3, 2]. Cheeger's problem is also tightly related to the continuous max-flow/min-cut duality [36, 37]. Finally, the Cheeger constant may also be viewed as the first eigenvalue of the 1-Laplacian $[17,28]$. In the case where $\Omega$ is convex, the recent papers $[10,1]$ prove uniqueness of the Cheeger set. Also, in two dimensions and when $\Omega$ is convex, an explicit construction for the Cheeger set is known (see [29]).

The connection between limit load analysis and the generalized Cheeger problem with weights (17) was established in [25, 22] which motivated the study of such weighted versions of Cheeger's problem. Various properties of the minimizers can be found in $[25,22,8]$. Existence of Cheeger sets for the weighted problem (17) follows from the direct methods of the calculus of variations but uniqueness does not hold in general which makes it difficult to compute numerically Cheeger sets. Fortunately, there is a unique maximal (for inclusion) Cheeger set (see [8]) and it is proved in [7] that it can be approximated by adding a small strictly convex penalization $\varepsilon \Phi$ to the original problem. More precisely, it is proved in [7] that the solution $u_{\varepsilon}$ of

$$
\sup _{u \in \mathrm{BV}, \pi(u) \leqslant 1} l(u)-\varepsilon \Phi(u)
$$

converges as $\varepsilon \rightarrow 0^{+}$to a multiple of the characteristic function of the maximal Cheeger set. Starting from this penalization scheme, with a quadratic $\Phi$, a convergent projection algorithm is implemented in [9] to approximate maximal Cheeger sets. Figure 1 shows example of maximal Cheeger sets approximated with this projection method.
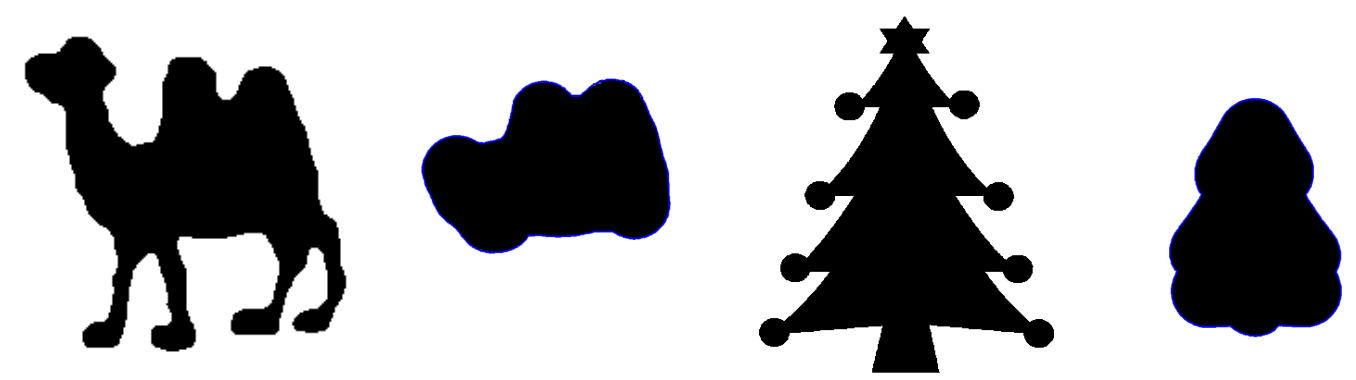

Figure 1: Example of $2 D$ domain $\Omega$ (left) and the corresponding maximal Cheeger sets (right), computed using the method of [9].

Our aim in the remainder of the paper is to generalize the strategy and the convergence results of [9] to handle the, physically more relevant, two-dimensional in-plane flow case that is detailed in the next paragraph. Of course, this two-dimensional extension presents several additional difficulties, both from a theoretical and numerical points, with respect to the unidimensional Cheeger case because of the incompressibility constraint on the one hand and the BD constraint on the other hand. 


\section{$3.2 \quad$ The in-plane case}

In order to state the limit load problem (14) for the in-plane flow case, let us set $\mathcal{D}=\Omega \times \mathbb{R}$ where $\Omega$ is a bounded domain in $\mathbb{R}^{2}$ with a smooth boundary $\partial \Omega$ divided into parts $\Gamma_{0}, \Gamma_{1}$ such that $\Gamma_{V}=\Gamma_{0} \times \mathbb{R}, \Gamma_{S}=\Gamma_{1} \times \mathbb{R}$. We are looking for a flow in the plane $O x_{1} x_{2}$, i.e. with $v_{3}=0$. We put $\boldsymbol{v}=(\boldsymbol{v}, 0)$ (independent of $\left.x_{3}\right)$ and we will use the same notation for the two components velocity field $v: \Omega \rightarrow \mathbb{R}^{2}$. In all examples we considered that the third components body and applied forces are vanishing $\left(b_{03}=f_{03}=0\right)$. We again use the notations $\nabla \boldsymbol{v}$ and $\boldsymbol{D}(\boldsymbol{v})$ for the two dimensional gradient operator of a two-dimensional vector field $\boldsymbol{v}=\left(v_{1}, v_{2}\right)$ and for its symmetric part

$$
\boldsymbol{D}(\boldsymbol{v})=\left(\begin{array}{cc}
\partial_{1} v_{1} & \frac{1}{2}\left(\partial_{1} v_{2}+\partial_{2} v_{1}\right) \\
\frac{1}{2}\left(\partial_{1} v_{2}+\partial_{2} v_{1}\right) & \partial_{2} v_{2}
\end{array}\right)
$$

The limit analysis problem (14) can then be rewritten as

$$
\frac{1}{\lambda}=\sup _{\boldsymbol{u} \in E, \Pi(\boldsymbol{u}) \leqslant 1} L(\boldsymbol{u}),
$$

where

$$
E:=\left\{\boldsymbol{u} \in \mathrm{BD}(\Omega) ; \operatorname{div}(\boldsymbol{u})=0 \text { in } \mathcal{D}^{\prime}(\Omega), \boldsymbol{u} \cdot \boldsymbol{n}=0 \text { on } \Gamma_{0}\right\},
$$

and the plastic power

$$
\Pi(\boldsymbol{u})=\int_{\Omega} \kappa(x) d|\boldsymbol{D}(\boldsymbol{u})|(x)+\int_{\Gamma_{0}} g|\boldsymbol{u}| .
$$

This variational problem has exactly the form studied in paragraph 2.2 for $d=2$. This the precisely this two-dimensional case that we will treat in our numerical simulations.

\section{Penalization scheme and convergence results}

\subsection{Convergence of the penalized scheme}

Our strategy to approximate numerically the relaxed form of the limit load problem $\left(\mathcal{P}_{0}\right)$ given by $(14)$ is simply to consider the following strictly concave perturbation of $\left(\mathcal{P}_{0}\right)$. For $\varepsilon>0$, let us consider the penalization

$$
\left(\mathcal{P}_{\varepsilon}\right) \sup \left\{L_{\varepsilon}(\boldsymbol{v}): \boldsymbol{v} \in E, \Pi(\boldsymbol{v}) \leqslant 1\right\}
$$

where

$$
L_{\varepsilon}(\boldsymbol{v}):=L(\boldsymbol{v})-\frac{\varepsilon}{d^{\prime}} \int_{\Omega}|\boldsymbol{v}|^{d^{\prime}}
$$

where $d^{\prime}=d /(d-1)$ is the conjugate exponent of the dimension $d$. Using both the density result of Proposition 1 (both for the intermediate and strong $L^{d^{\prime}}$ topology), we deduce that the maximum in $\left(\mathcal{P}_{\varepsilon}\right)$ is also the supremum when one restricts the maximization of $L_{\varepsilon}$ to more regular test-functions:

$$
\sup \left(\mathcal{P}_{\varepsilon}\right)=\sup \left\{L_{\varepsilon}(\boldsymbol{v}): \boldsymbol{v} \in E \cap C^{1} \cap \operatorname{LD}, \Pi(\boldsymbol{v}) \leqslant 1\right\} .
$$

Then, one easily gets the following:

Theorem 2. Under the same assumptions as in paragraph 2.2, one has: 
1. the set $\mathcal{S}$ of solutions of $\left(\mathcal{P}_{0}\right)$ is a nonempty convex and closed subset of $L^{d^{\prime}}$,

2. for every $\varepsilon>0,\left(\mathcal{P}_{\varepsilon}\right)$ admits a unique solution $\boldsymbol{u}_{\varepsilon}$,

3. $\left(\boldsymbol{u}_{\varepsilon}\right)_{\varepsilon}$ converges weakly in $\mathrm{BD}$ and strongly in $L^{d^{\prime}}$ to $\overline{\boldsymbol{u}}$ the element of $\mathcal{S}$ with minimal $L^{d^{\prime}}$ norm as $\varepsilon \rightarrow 0^{+}$.

Proof. 1. Nonemptyness of $\mathcal{S}$ has already been proved in theorem 1. The fact that $\mathcal{S}$ is convex follows from the concavity properties of problem $\left(\mathcal{P}_{0}\right)$ and the fact that it is closed in $\left.L^{d /(d-1}\right)$ follows again from the weak compactness in BD of the admissible set of $\left(\mathcal{P}_{0}\right)$ and the lower semicontinuity properties described above.

2. Existence for $\left(\mathcal{P}_{\varepsilon}\right)$ is exactly as in theorem 1 and uniqueness follows from the strict concavity of $L_{\varepsilon}$.

3. Since $\Pi\left(\boldsymbol{u}_{\varepsilon}\right) \leqslant 1$ for every $\varepsilon>0$, the family $\left(\boldsymbol{u}_{\varepsilon}\right)_{\varepsilon}$ is bounded in BD (and thus also in $\left.L^{\left.d^{\prime}\right)}\right)$. Let then $\varepsilon_{k} \rightarrow 0$ as $k \rightarrow+\infty$ be such that $\left(\boldsymbol{u}_{\varepsilon_{k}}\right)_{k}$ weakly converges in $L^{d^{\prime}}$ to some $\boldsymbol{u}$ as $k \rightarrow \infty$. Since for every $\varepsilon>0$ and every $\boldsymbol{v} \in E$ such that $\Pi(\boldsymbol{v}) \leqslant 1$ one has

$$
L\left(\boldsymbol{u}_{\varepsilon}\right)-\frac{\varepsilon}{d^{\prime}}\left\|\boldsymbol{u}_{\varepsilon}\right\|_{L^{d^{\prime}}}^{d^{\prime}} \geqslant L(\boldsymbol{v})-\frac{\varepsilon}{d^{\prime}}\|\boldsymbol{v}\|_{L^{d^{\prime}}}^{d^{\prime}}
$$

taking $\varepsilon=\varepsilon_{k}$ and letting $k \rightarrow \infty$, we deduce that $\boldsymbol{u} \in \mathcal{S}$. Taking $\boldsymbol{v}=\overline{\boldsymbol{u}}$ in (21), we then get

$$
\|\overline{\boldsymbol{u}}\|_{L^{d^{\prime}}} \geqslant \liminf _{k}\left\|\boldsymbol{u}_{\varepsilon_{k}}\right\|_{L^{d^{\prime}}} \geqslant\|\boldsymbol{u}\|_{L^{d^{\prime}}}
$$

so that $\boldsymbol{u}=\overline{\boldsymbol{u}}$ and the convergence of $\boldsymbol{u}_{\varepsilon_{k}}$ is strong in $L^{d^{\prime}}$ since $L^{d^{\prime}}$ is uniformly convex. Finally, the whole family $\left(\boldsymbol{u}_{\varepsilon}\right)_{\varepsilon}$ converges to $\overline{\boldsymbol{u}}$ by standard uniqueness/compactness arguments.

In our numerical examples, we will consider the case $d=2$, in this case $d^{\prime}=2$ so that $\left(\mathcal{P}_{\varepsilon}\right)$ simply amounts to project for the $L^{2}$ norm $\left(\boldsymbol{b}_{0}+\nabla f_{0}\right) / \varepsilon$ onto the closed and convex subset of $L^{2}, K:=\{\boldsymbol{v} \in E, \Pi(\boldsymbol{v}) \leqslant 1\}$. In this case, we then simply have

$$
\boldsymbol{u}_{\varepsilon}=\operatorname{proj}_{K}\left(\frac{\boldsymbol{b}_{0}+\nabla f_{0}}{\varepsilon}\right) .
$$

Of course, the $L^{2}$ projection is easier to handle than the $L^{d^{\prime}}$ one in general and this is why we will focus on the two-dimensional case in the sequel (although the penalization approach developed here also works in higher dimensions).

\subsection{Convergence for the discretized problems}

The aim of this paragraph is to prove, in dimension 2 and for fixed $\varepsilon>0$, the convergence of the solution of the discretization of the penalized problem $\left(\mathcal{P}_{\varepsilon}\right)$ to the solution of $\left(\mathcal{P}_{\varepsilon}\right)$. As shown above, in dimension $2,\left(\mathcal{P}_{\varepsilon}\right)$ is the $L^{2}$ projection problem:

$$
\inf _{u \in K}\left\|\boldsymbol{u}-\boldsymbol{u}^{0}\right\|_{L^{2}}
$$

where

$$
K:=\{\boldsymbol{u} \in E: \Pi(\boldsymbol{u}) \leqslant 1\}
$$

and $\boldsymbol{u}^{0}$ is related to the body forces, the normal traction and the penalization parameter $\varepsilon$ via

$$
\boldsymbol{u}^{0}=\frac{\boldsymbol{b}_{0}+\nabla f_{0}}{\varepsilon}
$$


In this paragraph, the form of $\boldsymbol{u}^{0}$ is not important, and one should rather think as $\boldsymbol{u}^{0}$ as an arbitrary $L^{2}$ vector field. The solution of (22) is denoted $\operatorname{proj}_{K}\left(\boldsymbol{u}^{0}\right)$.

For the sake of simplicity, we assume that $\Gamma_{V}=\emptyset$ (i.e. there is no Dirichlet boundary condition) and thus that the linear form $L$ vanishes on rigid motions. For mesh size $h>0$, we discretize the smooth two-dimensional domain $\mathcal{D}$ as follows. Denote by $\mathcal{D}^{h}$ the polyhedral domain whose closure is the union of all square cells $C_{i j}^{h}=[i h,(i+1) h] \times$ $[j h,(j+1) h]\left(\right.$ with $\left.(i, j) \in \mathbb{Z}^{2}\right)$ that intersect $\overline{\mathcal{D}}$. We then set

$$
I_{h}:=\left\{(i, j) \in \mathbb{Z}^{2}: C_{i j}^{h} \cap \overline{\mathcal{D}} \neq \emptyset\right\},
$$

and

$$
I_{h}^{\mathcal{D}}:=\left\{(i, j) \in I_{h}: C_{i j}^{h}, C_{(i-1) j}^{h}, C_{i(j-1)}^{h} \subset \mathcal{D}\right\} .
$$

Let $F_{h}$ denote the vector space $\left(\mathbb{R}^{2}\right)^{I_{h}^{\mathcal{D}}}$. For $\boldsymbol{u}=\left(u_{i j}^{1}, u_{i j}^{2}\right)_{i j} \in F_{h}$, partial derivatives of $\boldsymbol{u}$ are simply discretized by finite differences

$$
\partial_{1}^{h} \boldsymbol{u}_{i j}=\frac{1}{h}\left(\boldsymbol{u}_{i j}-\boldsymbol{u}_{(i-1) j}\right), \partial_{2}^{h} \boldsymbol{u}_{i j}=\frac{1}{h}\left(\boldsymbol{u}_{i j}-\boldsymbol{u}_{i(j-1)}\right),
$$

as well as the divergence operator

$$
\operatorname{div}^{h}(\boldsymbol{u})_{i j}:=\partial_{1}^{h} u_{i j}^{1}+\partial_{2}^{h} u_{i j}^{2},
$$

and the symmetrized gradient matrix

$$
\boldsymbol{D}^{h}(\boldsymbol{u}):=\frac{1}{2}\left(\partial_{k}^{h} \boldsymbol{u}^{l}+\partial_{l}^{h} \boldsymbol{u}^{k}\right)_{1 \leqslant k, l \leqslant 2} .
$$

Let us then define

$$
E_{h}:=\left\{\boldsymbol{u} \in F_{h}: \operatorname{div}^{h}(\boldsymbol{u})_{i j}=0, \forall(i, j) \in I_{h}^{\mathcal{D}}\right\} .
$$

Finally, let us define, for every $\boldsymbol{u} \in F_{h}$ :

$$
\begin{aligned}
\Pi_{h}(\boldsymbol{u}) & :=h^{2} \sum_{(i, j) \in I_{h}^{\mathcal{D}}} \kappa_{i j}\left|\boldsymbol{D}^{h}(\boldsymbol{u})_{i j}\right| \\
J_{h}(\boldsymbol{u}) & :=h^{2} \sum_{(i, j) \in I_{h}^{\mathcal{D}}}\left|\boldsymbol{u}_{i j}-\boldsymbol{u}_{i j}^{0}\right|^{2}
\end{aligned}
$$

and

$$
K_{h}:=\left\{\boldsymbol{u} \in E_{h}: \Pi_{h}(\boldsymbol{u}) \leqslant 1\right\}
$$

where $\kappa_{i j}$, and $\boldsymbol{u}_{i j}^{0}$ stand respectively for some discrete approximations (by mean values, say) of the data $\kappa$, and $\boldsymbol{u}^{0}$ respectively. The discretization of (22) then reads as

$$
\inf _{\boldsymbol{u} \in K_{h}} J_{h}(\boldsymbol{u})
$$

Let then $\boldsymbol{u}^{h}$ be the unique solution of the discretized projection problem (26) and, slightly abusing notations we shall also denote by $\boldsymbol{u}^{h}$ the piecewise constant vector field with value $\boldsymbol{u}_{i j}^{h}$ on the interior of the cell $C_{i j}^{h}$. The convergence of this scheme is then given by

Theorem 3. As $h \rightarrow 0^{+}, \boldsymbol{u}^{h}$ converges strongly in $L^{2}$ and weakly in $\mathrm{BD}$ to $\operatorname{proj}_{\mathrm{K}}\left(\boldsymbol{u}^{0}\right)$, the solution of (22). 
Proof. Since $\boldsymbol{u}^{h}$ is easily seen to be bounded in BD, it possesses a (not relabeled) subsequence that converges weakly in $\mathrm{BD}$ to some limit $\boldsymbol{u}$. Let us show that $\boldsymbol{u} \in K$ i.e.

$$
\operatorname{div}(\boldsymbol{u})=0, \text { and } \Pi(\boldsymbol{u}) \leqslant 1 .
$$

Let $\varphi \in C_{c}^{1}(\mathcal{D})$, setting $\varphi_{i j}:=\varphi(i h, j h)$, we first have

$$
\int_{\mathcal{D}} \nabla \varphi \cdot \boldsymbol{u}=\lim _{h} \sum_{(i, j) \in I_{h}^{\mathcal{D}}} h\left(\left(u^{1}\right)_{i j}^{h}\left(\varphi_{i+1, j}-\varphi_{i j}\right)+\left(u^{2}\right)_{i j}^{h}\left(\varphi_{i, j+1}-\varphi_{i j}\right)\right)
$$

and for $h$ small enough, since $\varphi$ has compact support in $\mathcal{D}$ and since $u^{h} \in E_{h}$, the latter sum equals

$$
-h \sum_{(i, j) \in I_{h}^{\mathcal{D}}} \operatorname{div}_{h}\left(\boldsymbol{u}^{h}\right)_{i j} \varphi_{i j}=0
$$

so that $\operatorname{div}(\boldsymbol{u})=0$. To prove that $\Pi(\boldsymbol{u}) \leqslant 1$, we use the representation formula

$$
\Pi(\boldsymbol{u})=\sup \left\{\int_{\mathcal{D}} \operatorname{div}(\boldsymbol{\sigma}) \cdot \boldsymbol{u}: \boldsymbol{\sigma} \in C_{c}^{1}\left(\mathcal{D}, \mathcal{S}_{2}\right),|\boldsymbol{\sigma}| \leqslant \kappa\right\}
$$

we then take $\boldsymbol{\sigma} \in C_{c}^{1}\left(\mathcal{D}, \mathcal{S}_{2}\right)$ such that $|\boldsymbol{\sigma}| \leqslant \kappa$ pointwise and discretize $\boldsymbol{\sigma}$ at points $(i h, j h)$ by values $\boldsymbol{\sigma}_{i j}$ such that $\left|\boldsymbol{\sigma}_{i j}\right| \leqslant \kappa_{i j}$, we then have

$$
\begin{aligned}
\int_{\mathcal{D}} \operatorname{div}(\boldsymbol{\sigma}) \cdot \boldsymbol{u} & =-\lim _{h} \sum_{(i, j) \in I_{h}^{\mathcal{D}}} h^{2} \boldsymbol{D}^{h}\left(\boldsymbol{u}^{h}\right)_{i j}: \boldsymbol{\sigma}_{i j} \\
& \leqslant \limsup \sum_{(i, j) \in I_{h}^{\mathcal{D}}} h^{2} \kappa_{i j}\left|\boldsymbol{D}^{h}\left(\boldsymbol{u}^{h}\right)_{i j}\right| \\
& =\limsup \Pi_{h}\left(\boldsymbol{u}^{h}\right) \leqslant 1 .
\end{aligned}
$$

Which proves that $\boldsymbol{u} \in K$.

Now let $\boldsymbol{v}=\left(v_{1}, v_{2}\right) \in K \cap C^{1}(\mathcal{D}) \cap L D$ and define the discretization, $\boldsymbol{v}^{h}=\left(\left(v^{1}\right)^{h},\left(v^{2}\right)^{h}\right)$ by

$$
\left(v^{1}\right)_{i j}^{h}:=h^{-1} \int_{h[(i, j-1),(i, j)]} v_{1},\left(v^{2}\right)_{i j}^{h}:=h^{-1} \int_{h[(i-1, j),(i, j)]} v_{2}
$$

since $\boldsymbol{v}$ is divergence-free, its flux through $\partial C_{i j}^{h}$ is zero and then $\boldsymbol{v}^{h} \in E_{h}$. Let $\delta>0$ since $\boldsymbol{v} \in C^{1}(\mathcal{D}) \cap \mathrm{LD}$, it easily follows from Lebesgue's dominated convergence theorem that

$$
\lim _{h} \Pi_{h}\left(\boldsymbol{v}^{h}\right)=\Pi(\boldsymbol{v}) \leqslant 1
$$

hence $(1+\delta)^{-1} \boldsymbol{v}^{h} \in K_{h}$ i.e. is admissible for (26) for $h$ small enough. We thus have

$$
F_{h}\left(\boldsymbol{u}^{h}\right) \leqslant F_{h}\left(\frac{\boldsymbol{v}^{h}}{1+\delta}\right)
$$

letting $h \rightarrow 0^{+}$, we thus have

$$
\left\|\boldsymbol{u}-\boldsymbol{u}^{0}\right\|_{L^{2}} \leqslant\left\|\frac{\boldsymbol{v}}{1+\delta}-\boldsymbol{u}^{0}\right\|_{L^{2}}
$$

letting $\delta \rightarrow 0^{+}$, we deduce that

$$
\left\|\boldsymbol{u}-\boldsymbol{u}^{0}\right\|_{L^{2}} \leqslant\left\|\frac{\boldsymbol{v}}{1+\delta}-\boldsymbol{u}^{0}\right\|_{L^{2}}, \forall \boldsymbol{v} \in K \cap C^{1}(\mathcal{D}) \cap \mathrm{LD}
$$


and we thus conclude from proposition 1 that $\boldsymbol{u}=\operatorname{proj}_{\mathrm{K}}\left(\boldsymbol{u}^{0}\right)$. By standard compactness/uniqueness argument, the whole sequence $\left(\boldsymbol{u}^{h}\right)$ thus converges to $\operatorname{proj}_{K}\left(\boldsymbol{u}^{0}\right)$ weakly in BD. Finally, strong $L^{2}$ convergence comes from

$$
\left\|\boldsymbol{u}^{h}-\boldsymbol{u}^{0}\right\|_{L^{2}} \rightarrow\left\|\boldsymbol{u}-\boldsymbol{u}^{0}\right\|_{L^{2}} \text { as } h \rightarrow 0^{+}
$$

and the uniform convexity of $L^{2}$.

The case where $\Gamma_{V} \neq \emptyset$ is not covered by the previous convergence result. In this more general case, one has two extra difficulties : discretizing in a consistent way the boundary conditions and the density in energy of smooth functions (which was a crucial point in the previous proof). Again, when $\mathcal{D}$ is a rectangle and $\Gamma_{V}$ consists of its vertical boundary, these difficulties may be overcome.

\section{Numerical computation of limit load}

This section describes a numerical scheme to solve the discretized version (26) of the limit load approximation problem (22). This scheme solves an unconstrained dual optimization problem using an accelerated first order algorithm.

\subsection{Dual unconstrained problem}

The following proposition shows that the discretized projection $\boldsymbol{u}^{h}$ that solves (26) can be computed by solving an unconstrained dual optimization problem. This dual optimization extends the result of [19] that was used to compute the projection of an image on a total variation ball.

Theorem 4. The solution $\boldsymbol{u}^{h}$ of (26) satisfies

$$
\boldsymbol{u}^{h}=\boldsymbol{P}^{h}\left(\boldsymbol{u}^{0}-\boldsymbol{D}^{h *}\left(\boldsymbol{\sigma}^{h}\right)\right),
$$

where $\boldsymbol{\sigma}_{i j}^{h} \in \mathbb{R}^{2 \times 2}$ is a solution of

$$
\min _{\boldsymbol{\sigma}} J(\boldsymbol{\sigma})=\frac{1}{2}\left\|\boldsymbol{P}^{h}\left(\boldsymbol{u}^{0}-\boldsymbol{D}^{h *}(\boldsymbol{\sigma})\right)\right\|^{2}+\|\boldsymbol{\sigma}\|_{\infty}
$$

where $\boldsymbol{D}^{h *}$ is the operator dual to $\boldsymbol{D}^{h}$ defined in (23), $\boldsymbol{P}^{h}$ is the orthogonal projector on the space $E_{h}$ defined in (24), and the $\ell^{\infty}$ norm of a tensor field $\boldsymbol{\sigma}_{i j} \in \mathbb{R}^{2 \times 2}$ reads

$$
\|\boldsymbol{\sigma}\|_{\infty}=\max _{(i, j) \in I_{h}^{\mathcal{D}}} \kappa_{i j}^{-1}\left|\boldsymbol{\sigma}_{i j}\right| .
$$

Proof. Introducing dual variables $\boldsymbol{\sigma}_{i j} \in \mathbb{R}^{2 \times 2}$, one can write the indicator function of the BD unit ball as

$$
1_{\Pi_{h}(\cdot) \leqslant 1}(\boldsymbol{u})=\max _{\boldsymbol{\sigma}}\left\langle\boldsymbol{\sigma}, \boldsymbol{D}^{h} \boldsymbol{u}\right\rangle-\|\boldsymbol{\sigma}\|_{\infty}=\left\{\begin{array}{ccc}
0 & \text { if } & \Pi_{h}(\boldsymbol{u}) \leqslant 1, \\
+\infty & \text { otherwise }
\end{array},\right.
$$

where the discrete BD norm is defined in (25). This allows one to rewrite (26) as

$$
\max _{\boldsymbol{\sigma}}-\|\boldsymbol{\sigma}\|_{\infty}+\min _{\boldsymbol{u} \in E_{h}}\left\langle\boldsymbol{\sigma}, \boldsymbol{D}^{h} \boldsymbol{u}\right\rangle+\frac{1}{2}\left\|\boldsymbol{u}-\boldsymbol{u}^{0}\right\|^{2} .
$$


The inner minimization is solved using the projector $\boldsymbol{P}^{h}$ as

$$
\boldsymbol{u}^{h}=\underset{\boldsymbol{u} \in E_{h}}{\operatorname{argmin}}\left\langle\boldsymbol{\sigma}, \boldsymbol{D}^{h} \boldsymbol{u}\right\rangle+\frac{1}{2}\left\|\boldsymbol{u}-\boldsymbol{u}^{0}\right\|^{2}=\boldsymbol{P}^{h}\left(\boldsymbol{u}^{0}-\boldsymbol{D}^{h *} \boldsymbol{\sigma}\right),
$$

and

$$
\left\langle\boldsymbol{\sigma}, \boldsymbol{D}^{h} \boldsymbol{u}\right\rangle+\frac{1}{2}\left\|\boldsymbol{u}^{0}-\boldsymbol{u}\right\|^{2}=-\frac{1}{2}\left\|\boldsymbol{P}^{h}\left(\boldsymbol{u}^{0}-\boldsymbol{D}^{h *} \boldsymbol{\sigma}\right)\right\|^{2}+c
$$

where $c$ is a constant that does not depends on $\boldsymbol{\sigma}$. Combining equations (28) and (29) leads to the optimization (27).

\subsection{Dual minimization algorithm}

To ease notations, we drop the dependency on the grid size $h$ in the remaining part of the paper.

Several iterative first order schemes exist to minimize (27), which is a sum of a smooth functional $\left\|\boldsymbol{P}\left(\boldsymbol{u}^{0}-\boldsymbol{D}^{*} \boldsymbol{\sigma}\right)\right\|^{2}$ and a non-smooth functional $\|\boldsymbol{\sigma}\|_{\infty}$.

Proximal operator. The non-smooth part $\|\boldsymbol{\sigma}\|_{\infty}$ of the functional is regularized during the iteration of a first order scheme by computing the proximal operator, for some $\rho>0$,

$$
\operatorname{prox}_{\rho\|\cdot\|_{\infty}}(\boldsymbol{\sigma})=\underset{\tilde{\boldsymbol{\sigma}}}{\operatorname{argmin}} \frac{1}{2}\|\tilde{\boldsymbol{\sigma}}-\boldsymbol{\sigma}\|^{2}+\|\tilde{\boldsymbol{\sigma}}\|_{\infty} .
$$

Proposition 2 shows that the proximal operator defined in (30) is computed explicitly using a soft thresholding $S_{\lambda}$ for a well chosen value of $\lambda$.

Computing the precise value of $\lambda$ for a given matrix field $\boldsymbol{\sigma}_{i j} \in \mathbb{R}^{2 \times 2}$ requires the computation of $d_{0} \leqslant d_{1} \leqslant \ldots \leqslant d_{N-1}$ that orders the set of norms

$$
\left\{d_{t}\right\}_{t=0}^{\left|I_{h}^{\mathcal{D}}\right|}=\left\{\left|\boldsymbol{\sigma}_{i j}\right|\right\}_{(i, j) \in I_{h}^{\mathcal{D}}}
$$

and also the cumulated ordered norms

$$
D_{s}=\sum_{t=s+1}^{N-1} d_{t} .
$$

Proposition 2. For $\boldsymbol{\sigma}_{i j} \in \mathbb{R}^{2 \times 2}$, one has

$$
\operatorname{prox}_{\rho\|\cdot\|_{\infty}}(\boldsymbol{\sigma})=\boldsymbol{\sigma}-S_{\lambda}(\boldsymbol{\sigma})
$$

where

$$
S_{\lambda}(\boldsymbol{\sigma})_{i j}=\max \left(1-\frac{\lambda \kappa_{i j}}{\left|\boldsymbol{\sigma}_{i j}\right|}, 0\right) \boldsymbol{\sigma}_{i j}
$$

and $\lambda>0$ is computed as

$$
\lambda=d_{t}+\left(d_{t+1}-d_{t}\right) \frac{D_{t+1}-\rho}{D_{t+1}-D_{t}}
$$

where $d$ and $D$ are defined in (31) and (32), and where $t$ is such that $D_{t+1} \leqslant \rho<D_{t}$. 
Proof. The resolution of (30) is computed using the relationship between the proximal operator of the norm $\|\cdot\|_{\infty}$ and the projection on the dual norm $\|\cdot\|_{1}$, see [16],

$$
\operatorname{prox}_{\rho\|\cdot\|_{\infty}}(\boldsymbol{\sigma})=\boldsymbol{\sigma}-\operatorname{Proj}_{\|\cdot\|_{1} \leqslant \rho}(\boldsymbol{\sigma})
$$

where the $\ell^{1}$ norm is defined as

$$
\|\boldsymbol{\sigma}\|_{1}=\sum_{(i, j) \in I_{h}^{\mathcal{D}}} \kappa_{i j}\left|\boldsymbol{\sigma}_{i j}\right| .
$$

and where $\operatorname{Proj}_{\|\cdot\|_{1} \leqslant \kappa}(\boldsymbol{\sigma})$ is the orthogonal projection of $\boldsymbol{\sigma}$ onto the set $\left\{\boldsymbol{\sigma} \backslash\|\boldsymbol{\sigma}\|_{1} \leqslant \rho\right\}$

$$
\operatorname{Proj}_{\|\cdot\|_{1} \leqslant \rho}(\boldsymbol{\sigma})=\underset{\tilde{\boldsymbol{\sigma}},\|\tilde{\boldsymbol{\sigma}}\|_{1} \leqslant \rho}{\operatorname{argmin}}\|\boldsymbol{\sigma}-\tilde{\boldsymbol{\sigma}}\| .
$$

The projection $\operatorname{Proj}_{\|\cdot\|_{1} \leqslant 1}(\boldsymbol{\sigma})$ is computed by noticing that is the solution of a regularized minimization

$$
\operatorname{Proj}_{\|\cdot\|_{1} \leqslant \rho}(\boldsymbol{\sigma})=\underset{\tilde{\boldsymbol{\sigma}}}{\operatorname{argmin}} \frac{1}{2}\|\boldsymbol{\sigma}-\tilde{\boldsymbol{\sigma}}\|^{2}+\lambda\|\tilde{\boldsymbol{\sigma}}\|_{1} .
$$

for a well chosen value of $\lambda>0$.

As noticed for instance in [11] for wavelet thresholding, the solution of such a $\ell^{1}$ regularized is computed using a soft thresholding

$$
\operatorname{Proj}_{\|\cdot\|_{1} \leqslant 1}(\boldsymbol{\sigma})=S_{\lambda}(\boldsymbol{\sigma}) .
$$

One can then check that the value (34) of $\lambda$ ensure that $\left\|S_{\lambda}(\boldsymbol{\sigma})\right\|=\rho$.

Multi-step First Order Scheme Y. Nesterov in [34, 33] proposes a first order scheme to find a minimizer $\boldsymbol{\sigma}^{h}$ of functional of the form $J(\boldsymbol{\sigma})$ as defined in (27). It computes iterates $\boldsymbol{\sigma}^{(k)}$ that converges to a solution $\boldsymbol{\sigma}^{h}$ using a multi-steps generalized gradient descent that makes use of previous iterates $\boldsymbol{\sigma}^{(i)}, i<k$ to compute $\boldsymbol{\sigma}^{(k)}$.

For a restricted set of non-smooth functionals, such as the functional $J$ minimized in (27), he shows that this method reaches the energy decay lower bound

$$
J\left(\boldsymbol{\sigma}^{(k)}\right)-J\left(\boldsymbol{\sigma}^{\star}\right)=O\left(1 / k^{2}\right)
$$

achievable by first order schemes. This method have found many applications recently in image processing, see for instance [43, 19].

Table 1 details the steps of Nesterov scheme to minimize (27). The step size $\mu$ should satisfy $\mu<2 /\left\|\boldsymbol{D} \boldsymbol{D}^{*}\right\|$ for the method to converge. This algorithm can be seen as an extension of the algorithm of Fadili and Peyré [19] that computes the projection on a ball of image with bounded variation. Our algorithm consider instead vector fields with bounded deformation, and includes a divergence free constraint that makes the computations more difficult.

A slight generalization of the result in [19] shows that not only one have a fast decay (35) on the objective function $J$, but also on the primal iterates, which is much more interesting for our application.

Theorem 5. If $\mu<2 /\left\|\boldsymbol{D} \boldsymbol{D}^{*}\right\|$, the iterates $\boldsymbol{u}^{(k)}=\boldsymbol{P}\left(\boldsymbol{u}^{0}-\boldsymbol{D}^{*} \boldsymbol{\sigma}^{(k)}\right)$ computed with the algorithm of Table 1 satisfy

$$
\left\|\boldsymbol{u}^{(k)}-\boldsymbol{u}^{h}\right\|^{2}=O\left(1 / k^{2}\right)
$$

where $\boldsymbol{u}^{h}$ is the solution of (26). 


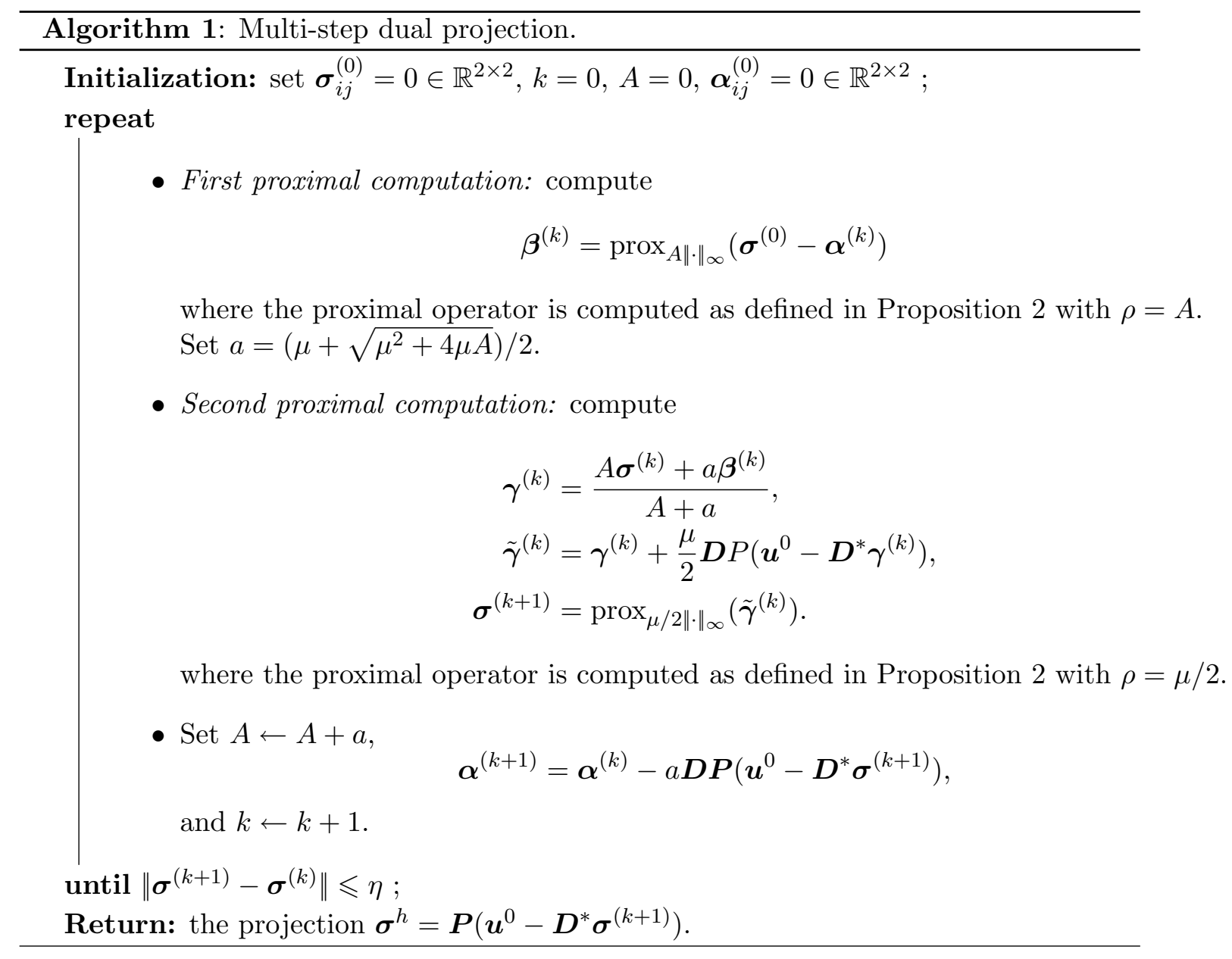


Proof. A similar result for the projection on the total variation ball has been proved in [19] (without linear constraints). The proof of [19] adapts directly to our situation.

The main computational bottleneck of the algorithm is the computation of the projection $\boldsymbol{P}$ on the set $E_{h}$ of divergence free vectors. In practice, this projection is computed approximately using a conjugate gradient descent. This projection should be computed with high enough precision to avoid convergences issue. In practice, one can use a warm restart and use the previous iterate as an initialization for the conjugate gradient, so that only a few steps are required at each iteration.

\section{$6 \quad$ Numerical results}

We present here some numerical simulations for three important limit load problems (two notched tensile, indentation and porous metals). For all numerical experiments, we have used a finite grid of $200 \times 200$ points, and the approximation parameter was chosen to be $\varepsilon=\Pi^{h}\left(\nabla f_{0}\right) / 100$.

\subsection{Two Notched Tensile Problem}

The two notched tensile problem is a popular benchmark test used for rigid-plastic and elasto-plastic analysis which was introduced by Nagtegaal et al. [32] to illustrate locking. The structure depicted in Figure 2 (left) consists of a rectangular specimen of length 2 with two thin notches under an in-plane tensile load $\tau_{0}$. Using the above notations we put $\Gamma_{0}=\emptyset, \Gamma_{1}=\partial \Omega$ and $\boldsymbol{f}_{0}=\left(0, \tau_{0}\right)$ on the right side, $\boldsymbol{f}_{0}=(0,-\kappa)$ on the left side. The yield limit is constant $\kappa=\sqrt{2} \tau_{0}$ and the body forces are vanishing $(\boldsymbol{b}=0)$, which means that $L(\boldsymbol{u})=\int_{\Gamma} \boldsymbol{f}_{0} \cdot \boldsymbol{u} d \Gamma$. We considered here only the case $a=1$.
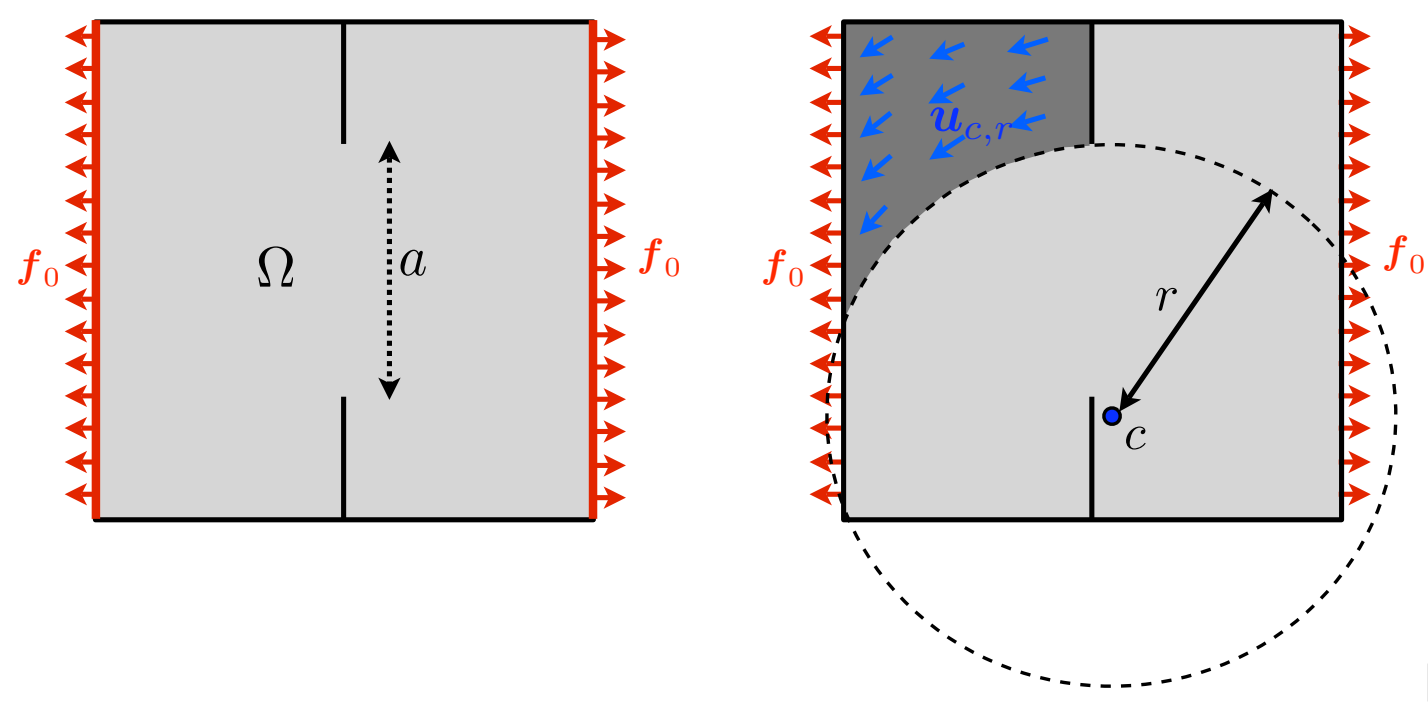

[ht]

Figure 2: Left: schematic view of the two notched tensile problem. Right: semi-analytical solution obtained with DVDS [26].

The "reference" value of is provided by Christiansen and Andersen [14], considered widely to be extremely accurate $\lambda \approx 1.136$. The same value was found by Ionescu and 
Oudet [26] with the discontinuous velocity domain splitting (DVDS) method. The corresponding fracture configurations are plotted in Figure 2 (right). DVDS uses a parametric family of vector field $\boldsymbol{u}_{c, r}$ parameterized by a center $c \in \mathbb{R}^{2}$ and a radius $r>0$, see an example in Figure 2, right. The vector field $\boldsymbol{u}_{c, r}$ corresponds to rotation movement outside the circle of center $c$ and radius $r$ and DVDS computes optimal parameters $(c, r)$ (see [26]) and they found

$$
\lambda \leqslant \lambda\left(\boldsymbol{u}_{c, r}\right)=\frac{\Pi\left(\boldsymbol{u}_{c, r}\right)}{L\left(\boldsymbol{u}_{c, r}\right)} \approx 1.136 .
$$

This result, obtained without any finite element discretization, is more accurate than $\lambda \approx 1.166$ obtained by Tin-Loi and Ngo [42] using a p-version finite element method $(p=15)$.

Figure 3, left, shows the solution $\boldsymbol{u}^{h}$ computed with our algorithm. The arrows shows some vectors $\boldsymbol{u}_{i j}^{h}$, and the color maps indexes the red (resp. blue) channel using the $\mathrm{X}$ (resp. Y) value of the vector field. Colors allows one to better visualize the discontinuities in the vector field. The numerical constant computed with our algorithm is

$$
\lambda\left(\boldsymbol{u}^{h}\right)=\frac{\Pi\left(\boldsymbol{u}^{h}\right)}{L\left(\boldsymbol{u}^{h}\right)} \approx 1.094 .
$$

This value is slightly smaller than the value computed in [26] and [14].

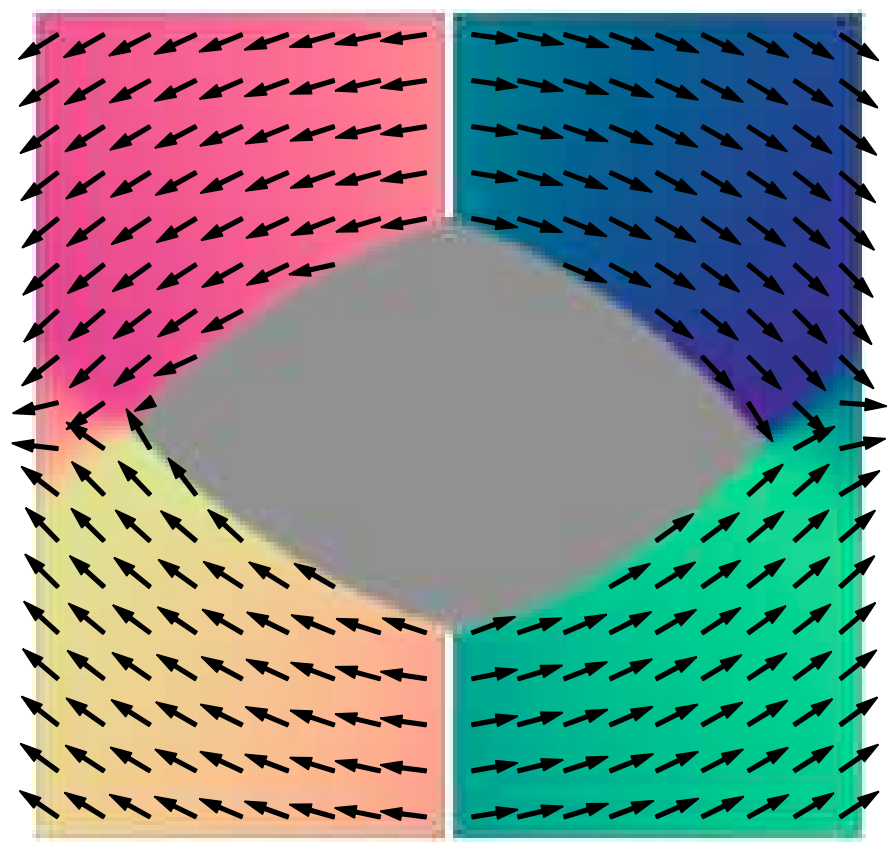

Figure 3: Numerical solution $\boldsymbol{u}^{h}$ computed with our algorithm for the two notched tensile problem.

\subsection{Indentation Problem}

In this problem, we consider a half space, that is handled numerically as an infinite rod with a elongated rectangle $\Omega$ section. The loading force $\boldsymbol{f}_{0}$ is localized on a small semgent $[A, B]$ of the upper boundary of the rod. Dirichlet condition $\boldsymbol{v}=0$ are imposed on the 
lower boundary $\Gamma_{V}$ of $\Omega$. The yield limit $\kappa=1$ is constant within the road. Figure 4 , left, shows the setting for this numerical experiment.

Two explicit vector fields $\boldsymbol{v}$ have been proposed by Hill and by Prandtl to find an upper estimation for $\lambda$ (see for instance [27]). They all give the same value

$$
\lambda \leqslant \lambda(\boldsymbol{v})=\frac{\Pi(\boldsymbol{v})}{L(\boldsymbol{v})}=\frac{2+\pi}{\sqrt{2}} \approx 3.64 .
$$

Figure 4, right, shows such an explicit solution, proposed by Prandlt (see [27]). It is constant over three orthogonal triangles $(A B E),(B C F),(C G D)$ and corresponds to a rotation over the two quarter of discs $(B E F),(C F G)$.
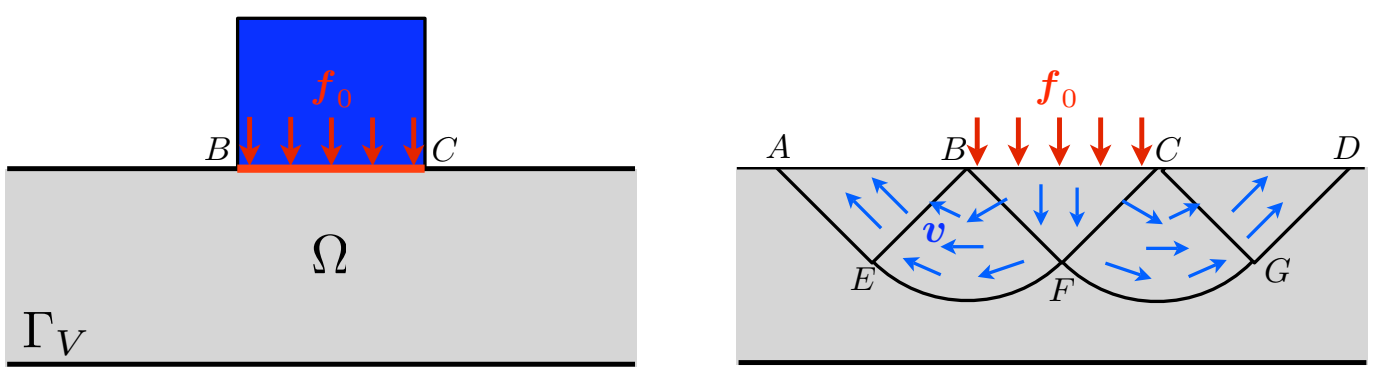

Figure 4: Left: schematic view of the indentation problem. Right: analytical solution of Prandlt.

Figure 5, right, shows the solution $\boldsymbol{u}^{h}$ computed with our algorithm. The numerical constant computed with our algorithm is

$$
\lambda\left(\boldsymbol{u}^{h}\right)=\frac{\Pi\left(\boldsymbol{u}^{h}\right)}{L\left(\boldsymbol{u}^{h}\right)} \approx 3.85 .
$$

This value is close but slightly larger than the value computed using the explicit solutions in (36), which shows that there is still room for improvements.

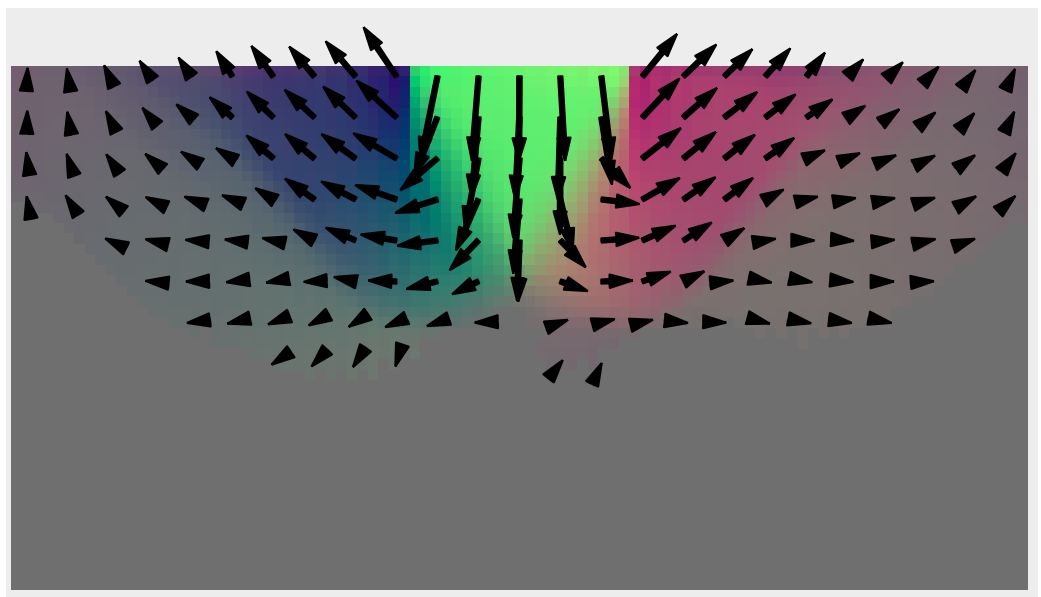

Figure 5: Numerical solution $\boldsymbol{u}^{h}$ computed with our algorithm for the indentation problem. 


\subsection{Compression of porous metals}

The ductile failure of porous metallic materials are usually studied using the lower and upper bound methods of limit load analysis. For cylindrical and spherical cavities, the problem wad firstly treated by Gurson with his famous kinematical approach [21], which gives an analytical upper bound of the homogenized yield criterion. There are lot of numerical approaches, using various limit load technics (see for instance [31, 20,6]) which confirmed and corrected the original Gourson model.

We want to illustrate here how our numerical method can be used to compute the limit load for metals with cavities. For that we consider $\Omega$ to be a square of unit side length, with a smaller square hole of side length $1 / 3$. The yield limit $\kappa=1$ is constant within $\Omega$ and the loading force (see Figure 6 left) is $\boldsymbol{f}_{0}=-\frac{1}{2} \boldsymbol{n}$ on the left and the right sides and $\boldsymbol{f}_{0}=-\frac{\sqrt{3}}{2} \boldsymbol{n}$, on the upper and bottom sides (here $\boldsymbol{n}$ is the exterior normal to the external boundary). In Figure 6 right we have plotted the numerical solution computed with our algorithm. We remark that the algorithm is able to capture the presence of two symmetric discontinuities(fractures) in the velocity field.
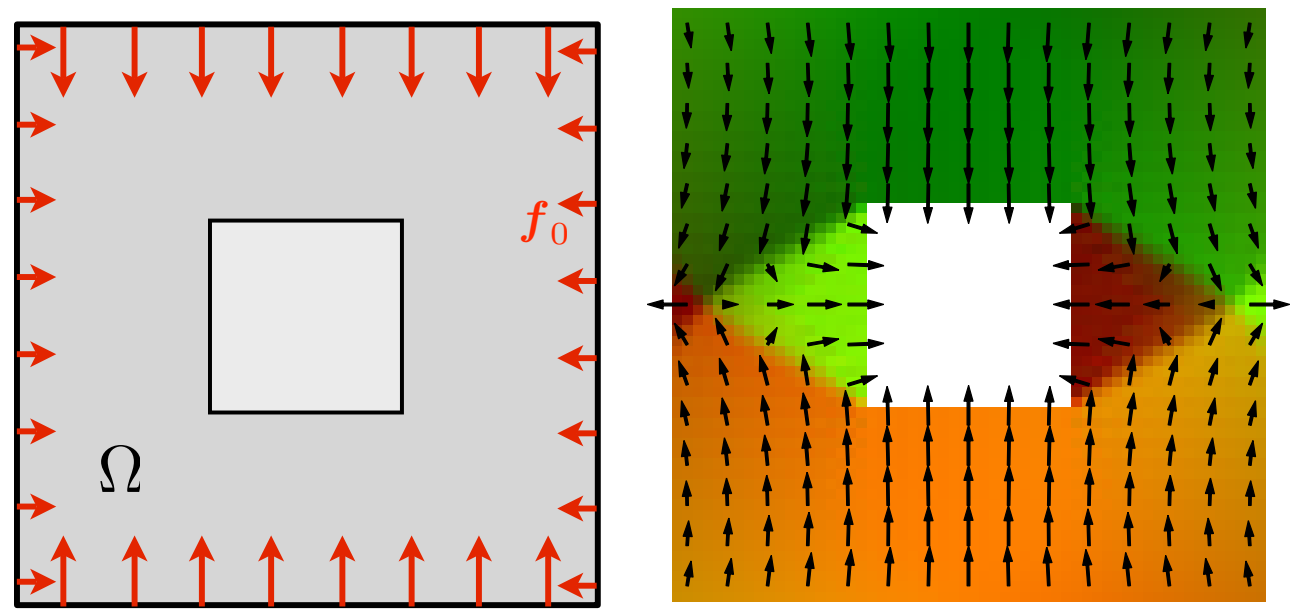

Figure 6: Left: plane-strain compression of a square with a square void. Right: numerical solution computed with our algorithm.

\section{Acknowledgments}

We would like to thank Jalal Fadili for his help during the development of the numerical scheme.

\section{References}

[1] F. Alter and V. Caselles. Uniqueness of the cheeger set of a convex body. Nonlinear Anal., 70(1):32-44, 2009.

[2] F. Alter, V. Caselles, and A. Chambolle. A characterization of convex calibrable sets in $\mathbb{R}^{n}$. Math. Ann., 332(2):329-366, 2005. 
[3] F. Alter, V. Caselles, and A. Chambolle. Evolution of characteristic functions of convex sets in the plane by the minimizing total variation flow. Interfaces Free Bound, $7(1): 29-53,2005$.

[4] K.D. Andersen, E. Christiansen, and M.L. Overton. Computing limit loads by minimizing a sum of norms. SIAM J. of Sci. Comp., 19(3):1046-1062, 1998.

[5] J.H. Argyris. Continua and discontinua. In Proceedings Conference on matrix methods in structural mechanics. Wright-Paterson AFB, 1967.

[6] N. Bilger, F. Auslender, M. Bornert, H. Moulinec, and A. Zaoui. Bounds and estimates for the effective yield surface of porous media with a uniform or a nonuniform distribution of voids. European Journal of Mechanics A/Solids, 26(5):810-836, 2007.

[7] G. Buttazzo, G. Carlier, and M. Comte. On the selection of maximal cheeger sets. Differential and Integral Equations, 20(9):991-1004, 2007.

[8] G. Carlier and M. Comte. On a weighted total variation minimization problem. $J$. Funct. Anal., 250(1):214-226, 2007.

[9] G. Carlier, M. Comte, and G. Peyré. Approximation of maximal cheeger sets by projection. ESAIM: Mathematical Modelling and Numerical Analysis, 43(1):131-150, 2009.

[10] V. Caselles, A. Chambolle, and M. Novaga. Uniqueness of the cheeger set of a convex body. Pacific J. Math., 232(1):77-90, 2007.

[11] A. Chambolle, R.A. De Vore, N. Lee, and B.J. Lucier. Nonlinear wavelet image processing: Variational problems, compression, and noise removal through wavelet shrinkage. IEEE Trans. Image Proc., 7(3):319-335, March 1998.

[12] J. Cheeger. A lower bound for the smallest eigenvalue of the laplacian. In Problems in Analysis, A Symposium in Honor of Salomon Bochner, pages 195-199, Princeton, 1970. Princeton Univ. Press.

[13] E. Christiansen. Limit analyis of collapse states. In P.G. Ciarlet and J.L. Lions, editors, Handbook of Numerical Analysis, volume 4, pages 193-312. Noth Holland, Amsterdam, 1996.

[14] E. Christiansen and K.D. Andersen. Computation of collapse states with von mises yield condition. International Journal for Numerical Methods in Engineering, 46(8):1185-1202, 1999.

[15] P.-L. Combettes and J.-C. Pesquet. Image restoration subject to a total variation constraint. IEEE Transactions on Image Processing, 13(9):1213-1222, 2004.

[16] P. L. Combettes and V. R. Wajs. Signal recovery by proximal forward-backward splitting. SIAM Journal on Multiscale Modeling and Simulation, 4(4), 2005.

[17] F. Demengel. On some nonlinear equation involving the 1-laplacian and trace map inequalities. Nonlinear Anal., Theory Methods Appl., 8(48):1151-1163, 2002.

[18] D. C. Drucker, W. Prager, and H.J. Greenberg. Extended limit design theorems for continuous media. Quarterly of Applied Mathematics, 9(4):381-389, 1952. 
[19] J. Fadili and G. Peyré. Total variation projection with first order schemes. Preprint Hal-00380491, 2009.

[20] P. Francescato, J. Pastor, and B. Riveill-Reydet. Ductile failure of cylindrically porous materials. part 1: Plane stress problem and experimental results. Eur. J. Mech. A/Solids, 23(2):181-190, 2004.

[21] A.L. Gurson. Continuum theory of ductile rupture by void nucleation and growth (part i - yield criteria and flow rules for porous ductile media). J. Eng. Mat. Tech., 99(1):2-15, 1977.

[22] R. Hassani, I.R. Ionescu, and T. Lachand-Robert. Shape optimization and supremal minimization approaches in landslides modeling. Applied Mathematics and Optimization, 52(3):349-364, 2005.

[23] D.G. Hayes and P.V. Marcal. Determination of upper bounds for problems in plane stress using finite element techniques. Internat. J. Mech. Scie., 9:245-251, 1964.

[24] P.G. Hodge and T. Belytschko. Numerical methods for the limit analysis of plates. J. Appl. Mech., 35:796-802, 1968.

[25] I. R. Ionescu and T. Lachand-Robert. Generalized cheeger sets related to landslides. Calc. Var. and PDE's, 23(2):227-249, 2005.

[26] I.R. Ionescu and E. Oudet. Discontinuous velocity domain splitting in limit analysis. submitted, 2009.

[27] L. Katchanov. Eléments de la théorie de la Plasticité. Edition Mir, 1973.

[28] B. Kawohl and V. Fridman. Isoperimetric estimates for the first eigenvalue of the $p$ laplace operator and the cheeger constant. Comm. Mat. Univ. Carol., 44(4):659-667, 2003.

[29] B. Kawohl and T. Lachand-Robert. Characterization of cheeger sets for convex subsets of the plane. Pacific J. Math., 225(1):103-118, 2006.

[30] K. Krabbenhoft, A. V. Lyamin, M. Hjiaj, and S. W. Sloan. A new discontinuous upper bound limit analysis formulation. International Journal for Numerical Methods in Engineering, 63(7):1069-1088, 2005.

[31] M.Trillat and J. Pastor. Limit analysis and gurson's model. European Journal of Mechanics A/Solids, 24(5):800-819, 2005.

[32] J.C. Nagtegaal, D.M. Parks, and J.R. Rice. On numerically accurate finite element solutions in the fully plastic range. Computer Methods in Applied Mechanics and Engineering, 4:153-177, 1974.

[33] Y. Nesterov. Smooth minimization of non-smooth functions. Math. Program., 103(1, Ser. A):127-152, 2005.

[34] Y. Nesterov. Gradient methods for minimizing composite objective function. CORE Discussion Papers 2007076, Université catholique de Louvain, Center for Operations Research and Econometrics (CORE), September 2007. 
[35] L.I. Rudin, S. Osher, and E. Fatemi. Nonlinear total variation based noise removal algorithms. Physica D, 60(1-4):259-268, 1992.

[36] G. Strang. Maximal flow through a domain. Math. Programming, 26(2):123-143, 1983.

[37] G. Strang. Maximum flows and minimum cuts in the plane. Advances in applied mathematics and global optimization, Adv. Mech. Math., 17:1-11, 2009.

[38] P. Suquet. Un espace fonctionnel pour les équations de la plasticité. Ann. Fac. Sci. Toulouse, V. Sér., Math., Sér. 5, 1(1):77-87, 1979.

[39] R. Temam. Problèmes mathématiques en plasticité. Gauthiers-Villars, Paris, 1983.

[40] R. Temam and G. Strang. Duality and relaxation in the variational problems of plasticity. J. de Mécanique, 19:493-527, 1980.

[41] R. Temam and G. Strang. Functions of bounded deformation. Arch. Ration. Mech. Anal., 75(1):7-21, 1980.

[42] F. Tin-Loi and N.S. Ngo. Performance of the p-version finite element method for limit analysis. International Journal of Mechanical Sciences, 45(6-7):1149-1166, 2003.

[43] P. Weiss, L. Blanc-Féraud, and G. Aubert. Efficient schemes for total variation minimization under constraints in image processing. SIAM Journal on Scientific Computing, 31(3):2047-2080, 2009. 